\title{
Noise-induced anti-correlated slow fluctuations in networks of neural populations
}

\author{
Dongmyeong Lee ${ }^{1}$, Seunghwan Kim ${ }^{1,2}$, Tae-Wook Ko ${ }^{3 *}$ \\ From Twenty Second Annual Computational Neuroscience Meeting: CNS*2013 \\ Paris, France. 13-18 July 2013
}

Coherent spontaneous fluctuations $(<0.1 \mathrm{~Hz})$ in fMRI blood-oxygen-level-dependent (BOLD) signal have been observed for a resting state of the human brain [1-4]. Functional connectivity analysis has identified clusters of brain areas exhibiting correlated fluctuations [1-4] and anti-correlation relationship between task-positive and task-negative areas [2-4]. In this study, we propose a model explaining the generation of slow fluctuations zand the organization of the clusters. Based on the slowness and the anti-correlation relationship, we describe the brain as a network of neural populations which act as brain areas and prefer one of the two states, UP (active) state and DOWN (quiescent) state [5], and consider excitation-inducing or inhibition-inducing connections between brain areas. Without noise, this system can have multiple stable states in which each area can be in UP, DOWN, or intermediate state. Presence of noise can make the system slowly move from one stable state to other and this is manifested as organized slow fluctuations. We implement this mechanism using a Wilson-Cowan model $[6,7]$ with excitatory and inhibitory neurons constituting the neural populations. The neural activity is translated into BOLD signal through the Balloon-Windkessel hemodynamic model $[8,9]$. With various networks with 2,3 , and 4 nodes, we show that the system without noise can have multiple stable states which are fixed points, and observe slow fluctuations and various organization including anti-correlated clusters. Similar behaviors are observed in the cases with random networks and modular networks. We analyze the functional connectivity in connection with the underlying networks.

\section{Acknowledgements}

DL and SK were supported by the Basic Science Research Program through the National Research Foundation of Korea (NRF) funded by the Ministry of Education, Science and Technology (No. 2012R1A1A2043674).

\section{Author details}

'Department of Physics, Pohang University of Science and Technology, Pohang 790-784, Republic of Korea. ${ }^{2}$ Institute for Edge of Theoretical Science, Pohang University of Science and Technology, Pohang 790-784, Republic of Korea. ${ }^{3}$ National Institute for Mathematical Sciences, Daejeon 305-811, Republic of Korea.

Published: 8 July 2013

\section{References}

1. Biswal B, Yetkin FZ, Haughton VM, Hyhe JS: Functional Connectivity in the Motor Cortex of Resting Human Brain Using Echo-Planar MRI. Magn Reson Med 1995, 34:537-541.

2. Fox MD, Snyder AZ, Vincent JL, Corbetta M, Van Essen DC, Raichle ME: The human brain is intrinsically organized into dynamic, anticorrelated functional networks. Proc Natl Acad Sci USA 2005, 102:9673-9678

3. Fox MD, Raichle ME: Spontaneous fluctuations in brain activity observed with functional magnetic resonance imaging. Nat Rev Neurosci 2007, 8:700-711

4. Deco G, Jirsa VK, McIntosh AR: Emerging concepts for the dynamical organization of resting-state activity in the brain. Nat Rev Neurosci 2011, 12:43-56.

5. Steriade M: Impact of Network Activities on Neuronal Properties in Corticothalamic Systems. J Neurophysiol 2001, 86:1-39.

6. Wilson HR, Cowan JD: Excitatory and inhibitory interactions in localized populations of model neurons. Biophys J 1972, 12:1-24.

7. Ermentrout GB, Terman DH: Mathematical Foundations of Neuroscience New York: Springer; 2010.

8. Friston KJ, Mechelli A, Turner R, Price CJ: Nonlinear responses in fMRI: the Balloon model, Volterra Kernels, and Other Hemodynamics. Neuroimage 2000, 12:466-477.

9. Friston K, Harrison L, Penny W: Dynamic causal modelling. Neuroimage 2003, 19:1273-1302.

doi:10.1186/1471-2202-14-S1-P214

Cite this article as: Lee et al: Noise-induced anti-correlated slow

fluctuations in networks of neural populations. BMC Neuroscience 2013 14(Suppl 1):P214.

\footnotetext{
* Correspondence: twko@nims.re.kr

${ }^{3}$ National Institute for Mathematical Sciences, Daejeon 305-811, Republic of Korea

Full list of author information is available at the end of the article
}

(c) 2013 Lee et al; licensee BioMed Central Ltd. This is an Open Access article distributed under the terms of the Creative Commons 